\title{
Evidencia basada en la práctica: conocimientos, percepciones y comportamiento de los estudiantes de primer año de Odontología
}

\author{
Evidence-based practice: knowledge, perceptions, and behavior of first year dental students
}

Sandra Lucia Montaño Rodríguez ${ }^{1, a}$, Martha Uzeda Salguero ${ }^{2, a}$, Limber Rodriguez Clavijo ${ }^{3, a}$

\section{Resumen}

La Odontología basada en la evidencia permite tomar decisiones clínicas fundamentadas en investigaciones actuales apoyadas en la mejor evidencia disponible, integrando la experiencia clínica con las preferencias y necesidades de los pacientes. Objetivo: el objetivo de este estudio fue determinar el estado inicial de los conocimientos, actitudes, acceso y confianza acerca de los principios de la Evidencia Basada en la Práctica de los estudiantes de primer año de la Facultad de Odontología de la Universidad Mayor de San Simón. Métodos: estudio descriptivo, prospectivo y transversal que aplicó el formulario KACE validado por Hendricson, que consta de 35 ítems dividido en cuatro áreas: Conocimientos, Actitudes, Acceso, Confianza. Resultados: los estudiantes participantes ( $\mathrm{n}=239$ ), presentaron en el Área Conocimiento $62,76 \%$; registraron respuestas correctas acerca del conocimiento de estudio de casos. Área Actitudes $67,37 \%$ indicó estar de acuerdo con la atención del paciente, basada en evidencia. Área Acceso a la Evidencia 40,17\%, de los estudiantes acceden a libros de texto en búsqueda de información; Área Confianza en las habilidades de evaluación crítica 45,61\% indicaron tener algo de confianza a acerca de la adecuación del tamaño de la muestra. Conclusión: de acuerdo a los resultados de este estudio, se debe profundizar la capacitación de conocimientos acerca de la Odontología Basada en la Práctica. En cuanto a actitudes los resultados muestran el interés de los estudiantes, en el área de acceso existen limitaciones, y acerca del área de confianza en las habilidades de evaluación crítica, los estudiantes registraron un valor regular.

Palabras clave: odontología basada en la práctica, odontología basada en evidencia, educación dental.

\section{Abstract}

Evidence-based Dentistry allows clinical decisions based on current research supported by the best available evidence, integrating the clinical experience, with the preferences and needs of the patients. Objective: the objective of this article was to determine the initial state of knowledge, attitudes, access, and confidence about the principles of Evidence Based on Practice of first-year students at Dental School at the San Simón University. Methods: descriptive, prospective cross-sectional study that applied the KACE form validated by Hendricson in 2012, consists of 35 items divided into four categories: Knowledge, Attitudes, Access, Confidence. Results: the students who participated $(n=239)$ registered in Knowledge category $62.76 \%$ correct answers about case study knowledge. In Attitudes category $67.37 \%$ indicated they agree with the evidence-based patient care. Access to Evidence category $40.17 \%$ of the students' access textbooks in search of information; Confidence category in critical evaluation skill $45.61 \%$ indicated having some confidence about the adequacy of the sample size. Conclusion: according to the results, the training of knowledge about Practice Based Dentistry should be deepened. Regarding attitudes, the results indicate student interest; in access to evidence, students registered a fair value in confidence in critical assessment.

Keywords: evidence-based dentistry, evidence-based practice, dental education.

$\mathrm{L}$ os programas de formación del área médica presentan una constante y rápida actualización como respuesta a las nuevas patologías emergentes a nivel local, nacional y mundial ${ }^{1-3}$. Estas actualizaciones deben seguir criterios de eficacia, como mencionó Archie Cochrane en su libro, en el año 1972: "Effectiveness and Efficiency: Random Reflections on Health Services".

En concordancia con esta premisa, años más tarde en 1992, Sacket y Guyatt ${ }^{5,6}$ de la Universidad de McMaster publicaron la Medicina Basada en Evidencia. Una nueva propuesta para

\footnotetext{
${ }^{1}$ Docente del Instituto de investigación, Odontología Social e Internado. aFacultad de Odontología, Universidad Mayor de San Simón, Cochabamba, Bolivia. https://orcid.org/0000-0002-5389-7486.

${ }^{2}$ Docente titular de la cátedra de Anatomía Humana.

3 Docente de la cátedra de Histología.

${ }^{*}$ Correspondencia a: Sandra Lucia Montaño Rodríguez

Correo electrónico: sandra_lumr@hotmail.com

Recibido el 15 de octubre de 2020. Aceptado el 12 de abril de 2021.
}

enseñar la práctica de la medicina.

En el año 2009, la Asociación Dental Americana (ADA) crea el Centro de Evidencia Basada en la Odontología, quienes definieron a la Odontología Basada en Evidencia ${ }^{5,7-10}$ como el «enfoque dirigido a la atención de la salud bucal que requiere la integración juiciosa de evaluaciones sistemáticas de evidencia científica clínicamente relevante, en relación a la condición, el historial médico y oral del paciente, la experiencia clínica del dentista, las necesidades y las preferencias de tratamiento del paciente».

A nivel europeo la Federación Mundial de Odontología - Región Europea (FDI- ERO) en forma conjunta con los Colegios profesionales de los Médicos Dentistas y las Universidades, creó en el año 2013 el proyecto: «Implementation of evidence-based dentistry into practice: análisis of qwareness, perceptions and attitudes of dentists in the World Dental Federation- European Region Organización zone» ${ }^{11}$, cuya finalidad fue la implementación 
de la Odontología Basada en Evidencias (OBE), actitudes, percepciones y nivel de conciencia de los dentistas con respecto a la OBE.

A nivel latinoamericano, las universidades crearon unidades específicas como la Unidad de Odontología Basada en Evidencia de la Universidad de Chile. Así mismo se realizaron formaciones en simposios, como es el caso de la Universidad Astral de Chile ${ }^{12}$. A su vez la Universidad de Sao Paulo, incluyó la Odontología Basada en Evidencia, tanto como una disciplina y/o como un área de enseñanza.

La enseñanza y aplicación de la Odontología Basada en Evidencia, responde a una necesidad social, la cual es la humanización de la ciencia al servicio de su comunidad, desde etapas tempranas de la formación profesional.

Acorde con estos lineamientos mencionados anteriormente Hendricson de la Universidad de Texas, en el año 2011 creó y validó un instrumento de evaluación para los elementos constitutivos de la OBE: el KACE (Knowledge, Attitudes, Access, Confidence $)^{13-16}$,(Conocimiento, Actitudes, Acceso y Confianza acerca de la evidencia. Estos ítems siguieron los lineamientos de Comisión de Acreditación Dental Americana $(\mathrm{CODA})^{17}$ en sus estándares de formación profesional en Odontología (Dental Education Program Standards) ${ }^{18}$.

El objetivo de este artículo fue determinar el estado inicial de los conocimientos, actitudes, acceso y confianza acerca de los principios de la Evidencia Basada en la Práctica (EBP) de los estudiantes de Primer año de la Facultad de Odontología de la Universidad Mayor de San Simón. Esta investigación forma parte del estudio: Odontología Basada en Evidencias, bioética y formación interprofesional su inserción transversal en la curricula pregradual de la Facultad de Odontología de la Universidad Mayor de San Simón.

\section{Material y métodos}

\section{Diseño de estudio y criterios de selección}

El diseño de este estudio fue descriptivo, transversal y prospectivo, realizado durante el segundo semestre de la gestión 2019, con la participación de los estudiantes de primer año de la Facultad de Odontología de la Universidad Mayor de San Simón, Cochabamba, Bolivia. Los criterios de inclusión fueron: a) estudiantes de primer año de la Facultad de Odontología, b) estudiantes que no hubiesen recibido capacitación previa acerca de Odontología Basada en Evidencia, c) estudiantes de ambos sexos, sin restricción de edad, d) estudiantes con acceso a correo electrónico. Los criterios de exclusión fueron: a) estudiantes de 2, 3, 4, 5 año de la facultad de Odontología, b) graduados, administrativos, docentes, c) estudiantes que no cumplan con los cuatro criterios de inclusión.

\section{Recolección de datos y herramientas de medición}

Se aplicó muestreo de tipo no probabilístico. Se solicitó a aquellos estudiantes que quisiesen participar en el estudio lo hicieran utilizando un link de acceso que se les proporcionó. Para proteger los datos personales, las encuestas fueron anónimas y se utilizó la aplicación de encuestas online de
Gmail. Los estudiantes no recibieron remuneración ninguna. De un total de 361 estudiantes matriculados en primer año participaron en este estudio 239 estudiantes.

Para el recojo de la información se utilizó la encuesta validada KACE por sus siglas en inglés (Knowledge, Attitudes, Access and Confidence Evaluation). La encuesta KACE con sus 35 ítems dividida en cuatro áreas: a) Conocimiento de la EBP (10 ítems), b) Actitudes acerca de la EBP (10 ítems), c) Acceso a la evidencia (9 ítems), d) Confianza en una evaluación crítica (6 ítems).

La aplicación del formulario KACE, fue autorizada por Hendrickson ${ }^{16}$. Se aplicó el formulario KACE con los mismos parámetros aplicados por Straub-Morarend y el consorcio de facultades norteamericanas ${ }^{14}$. La mencionada actualización fue traducida al español en su integridad, con los mismos términos, permitiendo que los parámetros de evaluación en el instrumento sean los mismos aplicados en otras facultades americanas. Por motivos de seguimiento y comparación del proceso se aplicó los porcentajes en las cuatro áreas. Los datos fueron analizados el programa SPSS v.26 IBM.

\section{Validez y fiabilidad del instrumento de medición}

La literatura científica dispone de diversos instrumentos, para evaluar la validez de los dominios de la Evidencia Basada en la Práctica ${ }^{19}$. El presente estudio aplica el KACE ${ }^{16,20,21}$, para evaluar el conocimiento inicial que tiene el estudiante de Odontología, sus comportamientos y habilidades de la EBP articuladas.

El desafío que todo cambio curricular basado en evidencia se plantea, es evaluar el antes y después de una formación académica. El KACE, permite la auto-evaluación del estudiante en su proceso formativo. Es verificable en forma práctica al final de la carrera como la capacidad de resolución de problemas basados en evidencia científica.

\section{Resultados}

Un total de 239 estudiantes participaron en este estudio. A los cuales se les preguntó acerca de cuatro áreas específicas de la Odontología Basada en la Práctica. Estas cuatro áreas fueron: el Conocimiento, Actitudes, Acceso y Confianza.

\section{Conocimiento de la evaluación crítica}

Los participantes registraron en el área de Conocimiento valores diferentes. De un total 239 estudiantes, 150 (62,76\%), estudiantes respondieron en forma correcta a la pregunta acerca de su conocimiento sobre estudios de caso. En cuanto a las respuestas acerca de los niveles de evidencia, $152(63,60 \%)$ estudiantes registraron respuestas incorrectas. Otro ítem registró un porcentaje elevado de respuestas incorrectas, fueron $163(68,20 \%)$ acerca de la pregunta PICO (Población, Intervención, Comparación, Resultado). Se observó que a partir de la sexta pregunta los porcentajes de las respuestas correctas fueron mayores en comparación de las cinco preguntas iniciales, cuyos porcentajes mayores estuvieron registrados en las respuestas incorrectas, con excepción de la pregunta 3, acerca de la búsqueda de información aplicando 
Tabla 1. Conocimientos Odontología Basada en Evidencia

\begin{tabular}{|c|c|c|c|}
\hline Ítem & $\begin{array}{l}\text { Respuestas } \\
\text { Correctas }\end{array}$ & $\begin{array}{l}\text { Respuestas } \\
\text { Incorrectas }\end{array}$ & «No sé» \\
\hline \multirow{2}{*}{ 1. Ranking de evidencia } & 33 & 152 & 54 \\
\hline & $13,81 \%$ & $63,60 \%$ & $22,59 \%$ \\
\hline \multirow{2}{*}{ 2. Valoración de la calidad de la literatura en Odontología } & 57 & 126 & 56 \\
\hline & $23,85 \%$ & $52,72 \%$ & $23,43 \%$ \\
\hline \multirow{2}{*}{$\begin{array}{l}\text { 3. Conocimiento de las estrategias de búsqueda de artículos } \\
\text { Científicos (PubMed/MeSH) }\end{array}$} & 101 & 95 & 43 \\
\hline & $42,26 \%$ & $39,75 \%$ & $17,99 \%$ \\
\hline \multirow{2}{*}{$\begin{array}{l}\text { 4. Conocimiento la importancia del número de sujetos en un } \\
\text { ensayo clínico }\end{array}$} & 71 & 105 & 63 \\
\hline & $29,71 \%$ & $43,93 \%$ & $26,36 \%$ \\
\hline \multirow{2}{*}{ 5. Conocimiento de la estructura PICO, para las investigaciones } & 29 & 163 & 47 \\
\hline & $12,13 \%$ & $68,20 \%$ & $19,67 \%$ \\
\hline \multirow[t]{2}{*}{ 6. Conocimiento acerca del estudio de caso en investigación } & 150 & 25 & 64 \\
\hline & $62,76 \%$ & $10,46 \%$ & $26,78 \%$ \\
\hline \multirow{2}{*}{ 7. Conocimiento acerca de Metaanálisis } & 141 & 27 & 71 \\
\hline & $59 \%$ & $11,30 \%$ & $29,70 \%$ \\
\hline \multirow{2}{*}{ 8. Conocimiento de como se realiza el diseño de una investigación } & 118 & 44 & 77 \\
\hline & $49,37 \%$ & $18,41 \%$ & $32,22 \%$ \\
\hline \multirow{2}{*}{ 9. Conocimiento acerca sensibilidad y especificidad } & 112 & 62 & 65 \\
\hline & $46,86 \%$ & $25,94 \%$ & $27,20 \%$ \\
\hline \multirow{2}{*}{ 10. Conceptualización de la prevalencia y la incidencia } & 102 & 74 & 63 \\
\hline & $42,68 \%$ & $30,96 \%$ & $26,36 \%$ \\
\hline
\end{tabular}

términos descriptivos en Medicina como es el MeSH PubMed en MEDLINE, los estudiantes respondieron 101 (42,26\%) de forma correcta (Tabla 1).

Actitudes con respecto a la práctica Basada en la Evidencia

El grupo de preguntas acerca de la variable actitudes en relación con la Odontología Basada en Evidencia (OBE), nos permite valorar la receptividad de los estudiantes hacia la $\mathrm{OBE}$, ya que está directamente relacionado con la valoración y significancia que el estudiante tiene con adoptar a la OBE en su práctica rutinaria.

De un total de 239 estudiantes, 116 (48,53\%) indicó que la práctica Basada en Evidencia es parte importante en la práctica profesional y $161(67,37 \%)$ estuvo de acuerdo con practicar la atención del paciente basada en la evidencia, 136 (56,90 \%) indicó estar de acuerdo en que la OBE puede cambiar la forma de aprendizaje. 102 (42,68\%) de los estudiantes consideran que la OBE es un «recetario» de atención dental ya que ignora la experiencia clínica (Tabla 2).

\section{Acceso a la Evidencia}

La tercera variable que analiza el KACE, es el acceso a la evidencia. A diferencia de las otras variables, las respuestas de los estudiantes no tuvieron consenso, ya que ninguna de las 8 preguntas de la variable acceso superaron en sus respuestas el $50 \%$.

Por lo que a continuación describimos los porcentajes mayores de cada ítem: $69(28,87 \%)$ de los estudiantes respondieron que acceden a menudo a la información de colegas, otros dentistas o proveedores de atención médica.
Los estudiantes respondieron que acceden a libros de texto en busca de información en forma frecuente, en un número de $96(40,17 \%)$.

Otra respuesta importante de tomar en cuenta fue que 74 $(30,7 \%)$ de los estudiantes acudían muy frecuentemente al internet. Con respecto al acceso a información de talleres de cursos de educación continua, a menudo son 87 (36,40\%) los estudiantes que buscan información en los mencionados talleres.

Con respecto al acceso a revistas indexadas, como la de Práctica Dental Basada en Evidencia o Medicina Basada en Evidencias, $72(30,12 \%)$ de los estudiantes indicaron que nunca accedieron. Acerca del acceso a la información de Podcast y conferencias web (webinar), 114 (47,5\%) de los estudiantes respondieron que nunca accedieron a esa fuente de capacitación (Tabla 3).

\section{Confianza en las habilidades de evaluación crítica}

Acerca de la variable confianza en las habilidades de evaluación crítica, los estudiantes respondieron en un nùmero de $93(38,91 \%)$ tener algo de confianza acerca de los diseños de estudio de las investigaciones.

En lo referente al sesgo en las investigaciones, $108(45,19$ $\%)$ de los estudiantes respondieron tener algo de confianza. En cuanto a la adecuación del tamaño de la muestra, 109 $(45,61 \%)$ estudiantes indicaron estar con algo de confianza. Con respecto al valor general del informe de investigación $85(35,3 \%)$ de los estudiantes indicaron tener algo de confianza (Tabla 4). 
Tabla 2. Actitudes acerca de la Odontología Basada en Evidencia

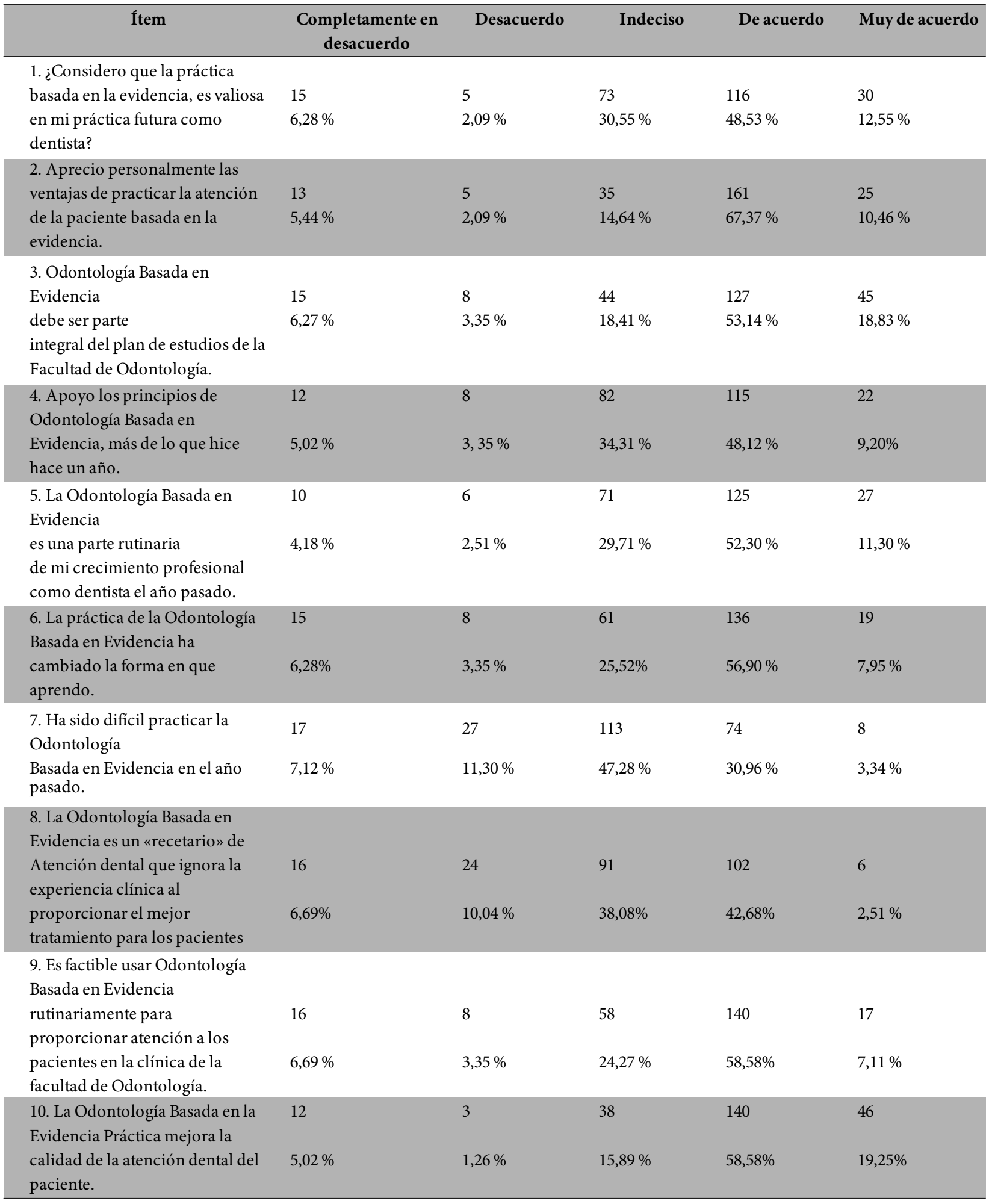

\section{Discusión}

La incorporación de la Odontología Basada en la Práctica ${ }^{13,22,23}$, en el diseño curricular de la carrera de Odontología ha constituido en los últimos años, un avance importante, en lo referente a cambios curriculares, ya que integran la teoría con la práctica, fundamentados en evidencia científica de calidad ${ }^{24}$.

Esta integración, conlleva la enseñanza y aprendizaje de competencias: cognitivas, procedimentales y actitudinales ${ }^{25,26}$, que partiendo del pensamiento crítico permiten al futuro 
Tabla 3. Acceso a la Evidencia

\begin{tabular}{|c|c|c|c|c|c|}
\hline Îtem & Nunca & Rara vez & Ocasionalmente & A menudo & Muy frecuente \\
\hline $\begin{array}{l}\text { 1. Acceso a información de } \\
\text { colegas, otros dentistas o } \\
\text { proveedores de atención } \\
\text { médica. }\end{array}$ & $\begin{array}{c}56 \\
23,43 \%\end{array}$ & $\begin{array}{c}63 \\
26,36 \%\end{array}$ & $\begin{array}{c}33 \\
13,81 \%\end{array}$ & $\begin{array}{cc}69 & \\
28,87 & \%\end{array}$ & $\begin{array}{c}18 \\
7,53 \%\end{array}$ \\
\hline $\begin{array}{l}\text { 2. Acceso a información de } \\
\text { libros de texto }\end{array}$ & $\begin{array}{c}6 \\
2,51 \%\end{array}$ & $\begin{array}{c}30 \\
12,55 \%\end{array}$ & $\begin{array}{c}41 \\
17,15 \%\end{array}$ & $\begin{array}{c}66 \\
27,62 \%\end{array}$ & $\begin{array}{c}96 \\
40,17 \%\end{array}$ \\
\hline $\begin{array}{l}\text { 3. ¿Acceso a Internet (excluidas } \\
\text { las revisiones Cochrane)? }\end{array}$ & $\begin{array}{c}16 \\
6,69 \%\end{array}$ & $\begin{array}{c}44 \\
18,41 \%\end{array}$ & $\begin{array}{c}52 \\
21,75 \%\end{array}$ & $\begin{array}{c}53 \\
22,3 \%\end{array}$ & $\begin{array}{c}74 \\
30,7 \%\end{array}$ \\
\hline $\begin{array}{l}\text { 4. ¿Acceso a un publicador } \\
\text { original de trabajos de } \\
\text { investigación en revistas } \\
\text { revisadas por pares? }\end{array}$ & $\begin{array}{c}63 \\
26,36 \%\end{array}$ & $\begin{array}{c}74 \\
30,96 \%\end{array}$ & $\begin{array}{c}34 \\
14,23 \%\end{array}$ & $\begin{array}{c}42 \\
17,57, \%\end{array}$ & $\begin{array}{c}26 \\
10,88 \%\end{array}$ \\
\hline $\begin{array}{l}\text { 5. ¿Acceso a la base de datos } \\
\text { Cochrane de revisiones } \\
\text { sistemáticas? }\end{array}$ & $\begin{array}{c}87 \\
36,40 \%\end{array}$ & $\begin{array}{c}66 \\
27,62 \%\end{array}$ & $\begin{array}{c}30 \\
12,55 \%\end{array}$ & $\begin{array}{c}40 \\
16,74 \%\end{array}$ & $\begin{array}{c}16 \\
6,69 \%\end{array}$ \\
\hline $\begin{array}{l}\text { 6. Acceso a la Revista de Práctica } \\
\text { Dental Basada en Evidencias o } \\
\text { Revista de Medicina Basada en } \\
\text { Evidencias? }\end{array}$ & $\begin{array}{c}72 \\
30,12 \%\end{array}$ & $\begin{array}{c}57 \\
23,85 \%\end{array}$ & $\begin{array}{c}31 \\
12,97 \%\end{array}$ & $\begin{array}{c}66 \\
27,62 \%\end{array}$ & $\begin{array}{c}13 \\
5,44 \%\end{array}$ \\
\hline $\begin{array}{l}\text { 7. Acceso a información de talleres } \\
\text { de cursos de educación } \\
\text { continua }\end{array}$ & $\begin{array}{c}59 \\
24,69 \%\end{array}$ & $\begin{array}{c}69 \\
28,87 \%\end{array}$ & $\begin{array}{c}7 \\
2,93 \%\end{array}$ & $\begin{array}{c}87 \\
36,40 \%\end{array}$ & $\begin{array}{c}17 \\
7,11 \%\end{array}$ \\
\hline $\begin{array}{l}\text { 8. Acceso a información de } \\
\text { Podcast y conferencias web } \\
\text { (webinars) }\end{array}$ & $\begin{array}{c}114 \\
47,5 \%\end{array}$ & $\begin{array}{c}56 \\
23,5 \%\end{array}$ & $\begin{array}{c}6 \\
2,5 \%\end{array}$ & $\begin{array}{c}54 \\
22,7 \%\end{array}$ & $\begin{array}{c}9 \\
3,8 \%\end{array}$ \\
\hline $\begin{array}{l}\text { 9. Acesso a Bases de datos de } \\
\text { temas evaluados criticamente } \\
\text { (CAT) }\end{array}$ & $\begin{array}{c}106 \\
44,35 \%\end{array}$ & $\begin{array}{c}55 \\
23,01 \%\end{array}$ & $\begin{array}{c}6 \\
2,51 \%\end{array}$ & $\begin{array}{c}65 \\
27,20 \%\end{array}$ & $\begin{array}{c}7 \\
2,93 \%\end{array}$ \\
\hline
\end{tabular}

profesional certeza en la toma de decisiones.

El pensamiento crítico promueve el debate y el juego de roles fortaleciendo las habilidades de comunicación ${ }^{27}$.Uno de los instrumentos de medición que considera al pensamiento crítico como esencial es el instrumento validado $\mathrm{KACE}^{13-15}$. El KACE, que ha sido ampliamente utilizado en los programas de cambios curriculares en las facultades de Odontología a nivel mundial ${ }^{20,23,28,29}$.

Las preguntas del área de conocimiento del KACE, concentra los elementos constitutivos de la EBP, en 10 preguntas, iniciando con el análisis del ranking de evidencia ${ }^{13}$, la calidad de literatura ${ }^{21}$, estrategias de búsqueda ${ }^{22}$, niveles de evidencia ${ }^{24,30}$, estructura PICO (Población, Intervención, Comparación, Resultado), características del ensayo clínico, diseño de las preguntas de investigación, diseño para evaluar la eficacia de los nuevos tratamientos y diferenciación entre incidencia y prevalencia ${ }^{31}$.

Los valores de las respuestas correctas, registradas en este estudio indican que, los estudiantes tienen una idea general de los principios de la investigación, siendo estas respuestas un aporte para la elaboración de la propuesta en el sentido de que se debe fortalecer la aplicabilidad de estos conocimientos generales para que se puedan elevar los porcentajes de las cinco primeras preguntas de está área.

El área de actitudes del formulario del KACE, permitió valorar la puesta en práctica de la $\mathrm{OBE}^{32}$. Las preguntas acerca de actitudes, proporcionaron parámetros de pronóstico, con respecto a la receptibilidad de la propuesta académica que incluye la Odontología Basada en Evidencia, ya que las preguntas contenidas en este grupo permiten evaluar el impacto de la OBE en la futura vida profesional, como son la toma de decisiones clínicas ${ }^{33}$ y resolución de problemas.

Los estudiantes participantes de este estudio registraron estar de acuerdo, acerca de las ventajas de practicar la atención del paciente basado en evidencia en la práctica rutinaria.

El área acceso a la evidencia, contemplado en el KACE, en la actualidad puede ser un factor limitante en el proceso enseñanza aprendizaje. Los datos registrados en este estudio, sugieren que es en el área de acceso, los valores con menor valor. Lo cual indica la falta o poco acceso a la evidencia.

La variable confianza en las habilidades de evaluación crítica, contempladas en el KACE, pretender fortalecer en el futuro profesional, la credibilidad de las estrategias de investigación y de sus componentes estructurales. 
Tabla 4. Confianza en las habilidades de evaluación crítica

\begin{tabular}{|c|c|c|c|c|c|}
\hline Ítem & $\begin{array}{l}\text { Nada de } \\
\text { confianza }\end{array}$ & $\begin{array}{l}\text { Algo de } \\
\text { confianza }\end{array}$ & $\begin{array}{l}\text { Moderadamente } \\
\text { seguro }\end{array}$ & Seguro & Confiado \\
\hline 1. ¿En su opinión son apropiados & 8 & 93 & 71 & 24 & 43 \\
\hline $\begin{array}{l}\text { los diseños de estudio de las } \\
\text { investigaciones? }\end{array}$ & $3,35 \%$ & $38,91 \%$ & $29,71 \%$ & $10,04 \%$ & $17,99 \%$ \\
\hline $\begin{array}{l}\text { 2. ¿Cual es su opinión sobre el } \\
\text { sesgo en el diseño del estudio o } \\
\text { análisis datos? }\end{array}$ & $\begin{array}{l}27 \\
11,30 \%\end{array}$ & $\begin{array}{l}108 \\
45,19 \%\end{array}$ & $\begin{array}{l}53 \\
22,18 \%\end{array}$ & $\begin{array}{l}16 \\
6,69, \%\end{array}$ & $\begin{array}{l}35 \\
14,64 \%\end{array}$ \\
\hline $\begin{array}{l}\text { 3. ¿Cual es su opinión sobre la } \\
\text { adecuación del tamaño de la } \\
\text { muestra? }\end{array}$ & $\begin{array}{l}22 \\
9,20 \%\end{array}$ & $\begin{array}{l}109 \\
45,61 \%\end{array}$ & $\begin{array}{l}51 \\
21,34 \%\end{array}$ & $\begin{array}{l}14 \\
5,86 \%\end{array}$ & $\begin{array}{l}43 \\
17,99 \%\end{array}$ \\
\hline $\begin{array}{l}\text { 4. ¿Cual es su opinión de la } \\
\text { Generalización de los } \\
\text { hallazgos? }\end{array}$ & $\begin{array}{l}20 \\
8,37 \%\end{array}$ & $\begin{array}{l}89 \\
37,24 \%\end{array}$ & $\begin{array}{l}67 \\
28,03 \%\end{array}$ & $\begin{array}{l}12 \\
5,02 \%\end{array}$ & $\begin{array}{l}51 \\
21,34 \%\end{array}$ \\
\hline $\begin{array}{l}\text { 5. ¿Que opina sobre el uso } \\
\text { apropiado de pruebas } \\
\text { estadísticas? }\end{array}$ & $\begin{array}{l}9 \\
3,8 \%\end{array}$ & $\begin{array}{l}92 \\
38,7 \%\end{array}$ & $\begin{array}{l}71 \\
29,8 \%\end{array}$ & $\begin{array}{l}22 \\
9,2 \%\end{array}$ & $\begin{array}{l}45 \\
18,5 \%\end{array}$ \\
\hline $\begin{array}{l}\text { 6. ¿Que opina del valor general } \\
\text { del informe de investigación? }\end{array}$ & $\begin{array}{l}15 \\
6,3, \%\end{array}$ & $\begin{array}{l}85 \\
35,3 \%\end{array}$ & $\begin{array}{l}51 \\
21,4 \%\end{array}$ & $\begin{array}{l}24 \\
10,1 \%\end{array}$ & $\begin{array}{l}64 \mathrm{~b} \\
26,9 \%\end{array}$ \\
\hline
\end{tabular}

Los estudiantes consultados con el formulario KACE, expresaron su poca confianza a las habilidades de evaluación crítica.

\section{Conclusión}

La aplicación del formulario validado KACE, permitió determinar de forma inicial la situación de los estudiantes de primer año de la Facultad de Odontología de la UMSS, con respecto a la Odontología Basada en Evidencia en la práctica y las cuatro áreas evaluadas: Conocimiento, Actitudes, Acceso y Confianza.

Este estudió concluye, que en el área Conocimiento, se deberá trabajar en forma independiente cada uno de los ítems y no así en forma grupal. En el área actitudinal, los resultados demuestran que existe motivación de los estudiantes con respecto a la Odontología Basada en la Práctica. Las dos áreas que en este estudio se sugiere mayor énfasis, son las de acceso y confianza en las habilidades de evaluación crítica.

Por tanto, este primer acercamiento a los parámetros internacionales de facultades de Odontología Americanas, Europeas y Latinoamericanas, permitirá en un futuro tanto acceso a evaluaciones externas por otras universidades, como evaluaciones internas al mismo proceso educativo de cambio curricular centrado en la incorporación de la Odontología Basada en Evidencia.

\section{Consideraciones Éticas}

El protocolo y formularios de recojo del estudio Odontología basada en evidencia, bioética y formación interprofesional su inserción transversal en la curricula pregradual de la Facultad de Odontología de la Universidad Mayor de San Simón, fue presentado al Comité de Bioética de la Universidad Mayor de San Simón.

\section{Agradecimientos}

Este trabajo no hubiese podido llevarse a cabo sin la colaboración desinteresada de: Dra. Jenny Rocabado, Dr. Victor Hugo Coca, Lic. Marco Paz, Lic. Hernan Rojas, Tec. Elizabeth Castro. Un agradecimiento especial a los Dres. William Hendricson, Teresa Marshall, Alonso Carrasco-Labra, Duarte Márques, Nataly Gantier-Limañi.

Conflicto de intereses: los autores declaran que no existe conflicto de intereses.

\section{Referencias bibliográficas}

1. van der Hoeven D, Hachem L El, Chaudhry S, Holland III JN, Shepherd KR. Methods and timing of curricular integration in U.S. dental education in preparation for the Integrated National Board Dental Examination. J Dent Educ [Internet]. 2020 Oct 8;n/a(n/a). Available from: https://doi. org/10.1002/jdd. 12442

2. Congdon HB. Interprofessional education (IPE) practices at universities across the United States with an established IPE infrastructure in place. J Interprofessional Educ Pract [Internet].
2016;5:53-8. Available from: http://dx.doi. org/10.1016/j.xjep.2016.10.001

3. Carrasco CGN, Gutiérrez LAV. Actualidad y perspectiva del currículum de odontología. Rev Cuba Educ Medica Super. 2011;25(4):515-24.

4. Trisha Greenhalgh. "Effectiveness and Efficiency: Random Reflections on Health Services.” Br Med J. 2004;328(7438):529.

5. American Dental Association, Alonso Carrasco-Labra, Romina Brignardello-Petersen, Michael Glick, Amir Azarpazhooh GG. How to
Use Evidence-Based Dental Practices to Improve Clinical Decision-Making.

6. Mckibbon A, Mckibbon A, Hunt D, Hunt D, Richardson WS, Richardson WS. Finding the evidence. In: Users Guides to the Medical Literature Essentials of Evidence- Based Clinical Practice. 2008. p. 33-76.

7. Abrahamyan L, Pechlivanoglou P, Krahn M, Carrasco-Labra A, Brignardello-Petersen R, Glick $M$, et al. A practical approach to evidencebased dentistry: IX: How to appraise and use 
an article about economic analysis. J Am Dent Assoc [Internet]. 2015;146(9):679-689. e1. Available from: http://dx.doi.org/10.1016/j. adaj.2015.06.021

8. Carrasco-Labra A, Brignardello-Petersen R, Glick M, Azarpazhooh A, Guyatt GH. How to use Evidence-Based Dental Practices to improve your Clinical Decision Making. ADA.American Dental Association., editor. Chicago, Illinois 60611: Perfect Bound Book; 2020. 299 p.

9. Carrasco-Labra A, Brignardello-Petersen R, Glick M, Guyatt GH, Neumann I, Azarpazhooh A. A practical approach to evidence-based dentistry: VII: How to use patient management recommendations from clinical practice guidelines. J Am Dent Assoc [Internet]. 2015;146(5):327-336.el. Available from: http:// dx.doi.org/10.1016/j.adaj.2015.03.015

10. Brignardello-Petersen R, CarrascoLabra A, Glick M, Guyatt GH, Azarpazhooh A. A practical approach to evidence-based dentistry: Understanding and applying the principles of EBD. J Am Dent Assoc [Internet]. 2014;145(11):1105-7. Available from: http:// dx.doi.org/10.14219/jada.2014.102

11. Yamalik N, Nemli SK, Carrilho E, Dianiskova $\mathrm{S}$, Melo P, Lella A, et al. Implementation of evidence-based dentistry into practice : analysis of awareness, perceptions and attitudes of dentists in the World Dental Federation - European Regional Organization zone *. 2015;127-45.

12. Uribe E. S. Qué es la odontología basada en la evidencia? Rev Fac Odontol Univ Valparaiso. 2000;(October 2000):281-7.

13. Cheryl L. Straub-Morarend, DDS, Christine R. Wankiiri-Hale, DMD, Derek R. Blanchette, MS, Sharon K. Lanning, DDS, Tanja Bekhuis, PhD, MS, MLIS, AHIP, Becky M. Smith, DDS, Abby J. Brodie, DMD, MS, Deise Cruz Oliveira, DDS, MS, MS, Robert A. Handy-sides, DD M (CIS) D. Evidence-Based Practice Knowledge, Perceptions, and Behavior: A Multi-Institutional, Cross-Sectional Study of Population of U.S Dental Students. J Dent Educ. 2015;2(2):147-85.

14. Straub-Morarend CL, Wankiiri-Hale CR, Blanchette DR, Lanning SK, Bekhuis T, Smith BM, et al. Evidence-Based Practice Knowledge, Perceptions, and Behavior: A Multi-Institutional, Cross-Sectional Study of a Population of U.S. Dental Students. J Dent Educ [Internet]. 2016;80(4):430-8. Available from: https://www. scopus.com/inward/record.uri?eid=2-s2.085016195519\&partnerID $=40 \& \mathrm{md} 5=\mathrm{b} 2946 \mathrm{ffb} 1 \mathrm{bf}$ 6ba4210cd5d1832d3189f

15. Rugh JD, Hendricson WD, Glass BJ et al. Teaching evidence-based practice at the University of Texas Health Science Center at San Antonio dental school. Vol. 128, Texas dental journal. 2011. p. 187-90.

16. Hendricson WD1, Rugh JD, Hatch JP, Stark DL, Deahl T WE. Validation of an Instrument to Assess Evidence-Based Practice (EBP) Knowledge, Attitudes, Access and Confidence. J Dent Educ. 2011;6(2):247-53.

17. Park, Sang E and Saldana, Fidencio and Donoff RB. A New Integrated Oral Health and Primary Care Education Program in the Dental Student Clinic. J Massachusetts Dent Soc [Internet]. 2016;64(4):26-30. Available from: http://europepmc.org/abstract/MED/27197363

18. Commission on Dental Accreditation. Commission on Dental Accreditation Accreditation Standards for Advanced Education Programs in General Practice Residency. 2015;

19. Shaneyfelt T, Baum KD, Bell D, Feldstein D, Houston TK, Kaatz S, et al. Instruments for evaluating education in evidence-based practice: A systematic review. J Am Med Assoc. 2006;296(9):1116-27.

20. Santiago V, Cardenas $M$, Charles $A L$, Hernandez E, Oyoyo U, Kwon SR. EvidenceBased Practice Knowledge, Attitude, Access and Confidence: A comparison of dental hygiene and dental students. J Dent Hyg JDH. 2018 Apr;92(2):31-7.

21. Parhar S, Gibson J. Does participation in an undergraduate journal club make dental students more knowledgeable and confident in practising evidence-based dentistry? Eur J Dent Educ Off J Assoc Dent Educ Eur. 2017 Nov;21(4):e59-63.

22. Kyriakoulis K, Patelarou A, Laliotis A, Wan AC, Matalliotakis M, Tsiou C, et al. Educational strategies for teaching evidence-based practice to undergraduate health students: systematic review. J Educ Eval Health Prof [Internet]. 2016;13:34. Available from: http://jeehp.org/ DOIx.php?id=10.3352/jeehp.2016.13.34

23. Stanley JL, Hanson CL, Van Ness CJ, Holt L. Assessing Evidence-Based Practice Knowledge, Attitudes, Access and Confidence Among Dental Hygiene Educators. J Dent Hyg JDH. 2015 Oct;89(5):321-9.
24. Oxford Centre for Evidence-based Medicine. Levels of Evidence. Univ Oxford [Internet] 2009;(November 1998):4-5. Available from: www. cebm.net

25. Beneitone Pablo, Esquetini César, Gonzãlez Julia, Maletá Maida Marty, Siufu Gabriela WR. Tunig America Latina. Reflexiones y perspectivas de la Educación Superior en América Latina. Deusto; 2007. 403 p.

26. Luis Eduardo González Fiegehen. Diseño Curricular Basado en Competencias y Aseguramiento de la Calidad de la Educación Superior. Centro Interuniversitario de Desarrollo CINDA, editor. 2016. 509 p.

27. Latif R, Mumtaz S, Mumtaz R, Hussain A. A comparison of debate and role play in enhancing critical thinking and communication skills of medical students during problem based learning. Biochem Mol Biol Educ a Bimon Publ Int Union Biochem Mol Biol. 2018 Jul;46(4):33642

28. Lallier TE. Introducing evidence-based dentistry to dental students using histology. J Dent Educ [Internet]. 2014;78(3):380-8. Available from: http://www.ncbi.nlm.nih.gov/ pubmed/24609340

29. Hinton RJ, Dechow PC, Abdellatif H, Mccann AL. Creating an Evidence-Based Dentistry Culture at Baylor College of Dentistry: The Winds of Change. J Dent Educ. 2011;75(3) (March):279-90.

30. Forrest JL. Introduction to the Basics of Evidence-Based Dentistry: Concepts and Skills. J Evid Based Dent Pract [Internet]. 2009;9(3):10812. Available from: http://dx.doi.org/10.1016/j. jebdp.2009.07.002

31. Petersen PE. The World Oral Health Report 2003: Continuous improvement of oral health in the 21st century - The approach of the WHO Global Oral Health Programme. Community Dent Oral Epidemiol. 2003;31(SUPPL. 1):3-24.

32. Wadgave U, Khairnar MR, Kadu TS, Chadha GK, Wadgave Y. Effect of training on evidencebased practice to undergraduate dental students: pre and postexperimental study. Int J Evid Based Healthc. 2020 Mar;18(1):101-7.

33. Elangovan S, Guzman-Armstrong S, Marshall TA, Johnsen DC. Clinical decision making in the era of evidence-based dentistry. Vol. 149, Journal of the American Dental Association. 2018. p. 745-7. 